\title{
VIDEOJUEGO EDUCATIVO COMO RECURSO PARA CONOCER Y PROTEGER ANIMALES EN PELIGRO DE EXTINCIÓN
}

\author{
EDUCATIONAL VIDEO GAME AS A RESOURCE TO SUPPORT TO KNOW AND \\ PROTECT ANIMALS IN DANGER OF EXTINCTION
}

\section{RICARDO ORTIZ1 , YEFFERSON TRELLES ${ }^{2}$, JOSÉ ORTIZ ${ }^{3}$}

1 Universidad Politécnica Territorial de Aragua “Federico Brito Figueroa”, Venezuela. raos.ortizsanchez@gmail.com

2 Universidad Politécnica Territorial de Aragua "Federico Brito Figueroa", Venezuela. yeferson26679880@gmail.com

3 Universidad de Carabobo, Venezuela. joseortiz.facesuc@gmail.com

RESUMEN

Se propone un videojuego educativo, como recurso de apoyo al aprendizaje de las Ciencias Naturales, del sexto grado de Educación Primaria. Se asume que el uso adecuado de tecnología en el aprendizaje contribuye a la comprensión y aplicación de los conceptos y propiedades vinculados con el medio ambiente. Como metodología se recurrió al proyecto factible. Para la elaboración de la propuesta se partió del diagnóstico de la situación resultado del trabajo de campo desarrollado en una Escuela Básica Nacional, del Estado Aragua (Venezuela). La información se obtuvo a través de una entrevista al docente de sexto grado de primaria y la aplicación de un cuestionario a los 22 alumnos cursantes del año escolar 2018-2019. La información recabada aportó una aproximación a la realidad del aprendizaje de la asignatura, los recursos utilizados para estudiar y las posibles necesidades de innovación. Este diagnóstico sirvió de base para diseñar la propuesta del videojuego educativo. El mismo fue diseñado en tecnología 3D, mediante el motor gráfico del software UNITY. Esta versión del videojuego permite que el estudiante interactúe con cinco animales que en Venezuela están en peligro de extinción, cada uno de hábitat diferente: halcón primito (desierto), oso frontino (bosque), tortuga carey (playa), caimán del Orinoco (río) y cachicamo (montaña). Adicionalmente, se aportan sugerencias y recomendaciones para mejorar el aprovechamiento del videojuego.

PALABRAS CLAVE: videojuego, preservación de la fauna, educación primaria.
An educational videogame is proposed, as a resource to support the learning of Natural Sciences, of the sixth grade of Primary Education. It is assumed that the appropriate use of technology in learning contributes to the understanding and application of concepts and properties related to the environment. The feasible project was used as a methodology. For the elaboration of the proposal, it was based on the diagnosis of the situation resulting from the field work developed in a National Basic School, of the Aragua State (Venezuela). The information was obtained through an interview with the sixth grade teacher and the application of a questionnaire to the 22 students attending the 2018-2019 school year. The information collected contributed an approximation to the reality of the subject's learning, the resources used to study and the possible innovation needs. This diagnosis served as the basis for designing the educational video game proposal. It was designed in 3D technology, through the graphic engine of the UNITY software. This version of the videogame allows the student to interact with five animals that are in danger of extinction in Venezuela, each of a different habitat: primitive hawk (desert), spectacled bear (forest), hawksbill turtle (beach), caiman of the Orinoco (river) and armadillo (mountain). Additionally, suggestions and recommendations are provided to improve the use of the videogame.

KEYWORDS: videogame, preservation of fauna, primary education. 
INTRODUCCIÓN

La UNESCO (1997) sostiene que la escuela primaria es el sitio más natural para incorporar a los niños a la educación ambiental. Esta formación temprana contribuye a la toma de conciencia y el conocimiento sobre hechos y relaciones de las personas con su medio ambiente, de tal manera que tiendan a mantener la vida digna en el planeta.

Los niños van a la escuela con muchas inquietudes y deseos de aprender. Eso representa un elemento favorable para el trabajo en el aula, que puede ser aprovechado por el docente para la incorporación de estrategias educativas atractivas, creativas y novedosas que involucren el proceso formativo con la imaginación del niño. Por lo tanto, en las aulas de las escuelas, los maestros deben estar dotados no solo de herramientas pertinentes, novedosas y versátiles, sino además contar con la preparación necesaria para interactuar con los alumnos y responder a sus expectativas. En ese sentido, las nuevas tecnologías tienen mucho que aportar tanto para el docente como para el alumno; por ejemplo, un maestro podría visitar un museo o un parque natural en cualquier parte del mundo, utilizando internet en el aula para presentar y explicar temas de ciencias naturales. Asimismo, un alumno podría explorar y desarrollar actividades de aprendizaje, que pueden ser compartidas vía internet, con estudiantes de su mismo grado, bien sea de la institución donde cursa estudios o de otras.

El uso del computador por parte del alumno, en sus múltiples posibilidades, podría aprovecharse para hacer tareas (dibujos, tablas, textos, videos, entre otras) y también para aprender jugando, tanto con juegos 2D como 3D y para propósitos específicos, tal como señalan Michael y Chen (2006), como es el caso de acciones de naturaleza académica orientadas al reforzamiento de contenidos, la incentivación al desarrollo de la curiosidad e indagación individual para complementar saberes.

Sin embargo, tanto de fuentes documentales (Boada y Escalona, 2004; Solano, Forero, Cavanzo y Pinilla, 2013), como de la experiencia con alumnos en el aula de sexto grado, se ha podido conocer que la educación ambiental se aprende, muchas veces, a partir de explicaciones orales del docente, sin acudir a otros recursos que contribuyan, por una parte, a consolidar los conocimientos sobre el tema ambiental y, por la otra, a despertar mayor interés por sus contenidos, tomando en cuenta la importancia que éstos tienen para la formación de ciudadanos respetuosos del medio ambiente. Es decir, en las escuelas públicas de Venezuela, aunque existe un programa oficial de dotación de computadoras Canaima para escolares, no se ha considerado la incorporación en el aula de recursos digitales como los videojuegos, para abordar los temas de la educación ambiental, a pesar que los alumnos por lo general poseen experiencia como usuarios de estos dispositivos y son particularmente sensibles e interesados en su uso, pues generalmente forman parte de su rutina diaria. Por el contrario, hay resistencia o actitud negativa de muchos maestros respecto al uso de los videojuegos educativos (López, 2016). No obstante, en numerosas investigaciones se presentan conclusiones favorables que reportan las virtudes del uso de éstos dispositivos en el aula por parte de los niños y niñas, adolescentes y educadores, en el proceso de enseñanza y aprendizaje (Escobar, 2017; Grande, 2018).

Este problema se ha abordado en China, Japón, Singapur, Estados Unidos, Finlandia y la mayor parte de Europa (Gee,2003; Çiftci,2017). En estos países utilizan los videojuegos educativos porque se ha demostrado que resulta una manera muy efectiva para la concentración y el aprendizaje de los niños, ya que es un mecanismo atractivo para interactuar en escenarios y ambientes creados para que los niños y niñas exploren. Esto se evidencia en el hecho que cada día aumentan en el mundo los usuarios de videojuegos tal como señalan publicaciones relacionadas con el tema (Malykhina, 2014).

En algunos países, como es el caso de Venezuela, aún no hay un desarrollo importante en el uso de videojuegos educativos. Los niños, niñas y jóvenes tienden a jugar videojuegos comerciales, los cuales no traen especificaciones educativas; es decir, están más centrados en el entretenimiento que en acciones directas articuladas a un objetivo educativo.

En el ámbito regional y local, se han ejecutado algunos proyectos del uso de juegos (no videojuegos), los cuales han sido publicados por la Universidad de Carabobo en su repositorio institucional, disponible en www.bc.uc.edu. ve. Lamentablemente, se echa en falta investigaciones relacionadas con los videojuegos educativos y específicamente en temas relacionados con la educación ambiental. Esto se constató al efectuar una indagación sistemática en varias bases de datos de investigación educativa, donde se encontró que hay pocos trabajos sobre el uso de videojuegos 3D en el aprendizaje de 
temas relacionados con la educación ambiental en particular.

Uno de los elementos que fundamenta el interés de la investigación sobre el tema es que muchos niños y niñas en la escuela primaria perciben el trabajo en el aula poco interesante, tedioso y no logran concentrarse en sus estudios o se aburren en las clases. Ante esta realidad, se parte del supuesto que aplicando estrategias novedosas en el aula, tales como los videojuegos, se podría reducir considerablemente el "fastidio" de ir a clases. Asimismo, no se debe olvidar mencionar que el niño y la niña en la actualidad viven en un mundo donde la tecnología reina en su ambiente, es parte de su cotidianidad. En este sentido, se considera importante incursionar en el diseño de videojuegos educativos, recurriendo a plataformas como Unity (Creighton, 2010), en tanto que ésta constituye una potente herramienta computacional para diseñar videojuegos educativos que resulten atractivos para los niños y niñas, en especial para temas tan sensibles como los contenidos en la educación ambiental.

La situación descrita pone en evidencia la necesidad de generar acciones concretas y factibles dirigidas a procurar, en el marco del aula escolar, actuaciones tangibles dirigidas a la sensibilización ante los problemas de la conservación ambiental, en particular en lo concerniente a la situación de los animales en peligro de extinción. La realidad de la problemática obliga a direccionar la mirada hacia el estudiante y despertar su interés hacia la ampliación de conocimientos sobre temas como la educación ambiental, los cuales le serán de gran utilidad para los grados inmediatamente superiores, además de concientizar la importancia de su rol como ciudadano en la conservación del ambiente. Es decir, la no actuación en el ámbito escolar, en tanto que etapa crucial en la formación de la conciencia ciudadana de respeto y conservación del equilibrio ambiental, haría prevalecer en los niños y niñas la falta de interés no solo hacia el estudio de los temas ambientales, sino además sobre la importancia que tiene para la vida la conservación del entorno natural. En atención a lo antes expuesto se diseña un videojuego educativo, bajo tecnología 3D, específicamente en lenguaje UNITY, para ser utilizado en el aprendizaje de conceptos ambientales, correspondientes al programa de educación primaria, de sexto grado, específicamente, la identificación y protección de los animales que en Venezuela, se encuentran en peligro de extinción.

Esta propuesta se soporta en la consideración que los videojuegos con propósitos específicos, en particular los educativos, permiten a los estudiantes vivir o imaginar situaciones que serían imposibles en el mundo real por razones de seguridad, economía, tiempo, entre otros (McFarlane, Sparrowhawk y Heald, 2002; Diaz Barriga, 2006). Además de tener un impacto positivo en el desarrollo intelectual de los jugadores (Michael y Chen, 2006), en el caso particular del videojuego propuesto, el mismo procura sensibilizar a los usuarios en torno a la conservación de los animales en peligro de extinción.

La UNESCO (2007), afirma que: La Educación Ambiental es un proceso que ayuda a desarrollar las habilidades y actitudes necesarias para comprender las relaciones entre los seres humanos, sus culturas y el mundo biofísico. Todo programa de educación ambiental (...) debería estimular la curiosidad, fomentar la toma de conciencia y orientar hacia un interés informado que eventualmente será expresado en términos de una acción positiva (p.4).

La cita anterior refleja la gran importancia dada a la educación ambiental por un organismo como la Organización de las Naciones Unidas para la Educación, la Ciencia y la Cultura (UNESCO). Esto significa que los distintos países que conforman esta organización, entre ellos Venezuela, deben tener directrices en ese sentido, para incorporar la educación ambiental en la escuela primaria. En ese sentido, es importante destacar que en Venezuela, en la actualidad el currículo de educación primaria, incluye los contenidos de educación ambiental en elárea de aprendizaje denominada "Matemática, Ciencias Naturales y Sociedad" (Ministerio del Poder Popular para la Educación, 2007). Es decir, se define la educación ambiental como un contenido asociado con otras temáticas curriculares que son de gran peso en la formación del niño, lo cual podría favorecer la interrelación disciplinar en beneficio de la consolidación de los conocimientos y sus implicaciones sobre la formación científica de los estudiantes (Vargas, 2016).

Es precisamente con el interés de estar a tono con los planteamientos de la UNESCO (2007), en lo referente al valor que tiene la Educación Ambiental, en tanto que espacio para a desarrollar en los niños y niñas la comprensión de la relación de los seres humanos con el mundo biofísi- 
co, que se diseñó un videojuego educativo, como herramienta de apoyo al proceso de enseñanza y aprendizaje de los contenidos ambientales en sexto grado. El mismo está orientado a dar respuesta a las deficiencias observadas en el aula de sexto grado, particularmente respecto a la no incorporación de recursos no tradicionales en el aula, situación que en su investigación Colmenares y Sánchez (2015) habían puesto en videncia. La carencia podría revertirse utilizando las TIC y en especial los videojuegos, los cuales tienen la capacidad de atraer a los estudiantes al maravilloso placer de aprender y a la resolución de problemas (Vargas, 2015, Vásquez, 2015).

Por otro lado, es importante acotar que además de generar un ambiente atractivo para los escolares, la interacción con el videojuego educativo favorece el desarrollo de habilidades digitales, trabajo en grupo, coordinación mano-vista, habilidades para tomar decisiones y desarrollo de la creatividad (Rojo y Dudu, 2017).

METODOLOGÍA

La presente investigación se enmarcó en la modalidad de proyecto factible, ya que se sostiene en el diseño de una propuesta de acción dirigida a resolver un problema o necesidad previamente detectada en el medio (Dubs, 2002; UPEL, 2016). El diagnóstico de la situación se efectuó a partir de un trabajo de campo, en la Escuela Básica Nacional "Aníbal Castillo", ubicada en el Barrio "23 de Enero", de la Ciudad de Maracay, Municipio Girardot, del Estado Aragua (Venezuela). Se consideró para el estudio la sección Única de sexto grado existente en la escuela. Participaron como informantes el maestro y los 22 estudiantes del curso académico 2018-2019. Para la recogida de la información se efectuó una entrevista al docente y se le aplicó un cuestionario a sus estudiantes. El cuestionario fue diseñado según los indicadores y dimensiones de las variables (Conocimiento en educación ambiental, Disposición de los alumnos hacia las innovaciones y El videojuego como recurso de aprendizaje) correspondientes. Asimismo, dicho cuestionario fue validado por panel de expertos, quienes dieron sus aportes para concluir en el cuestionario definitivo que fue aplicado, en primer lugar, a un grupo piloto, formado por seis estudiantes de sexto grado de escuelas públicas, lo cual resultó de mucha utilidad para la calibración de los ítems. Los aspectos recogidos en el cuestionario estaban dirigidos a satisfacer la fase del diagnóstico de la situación; en este sentido el mismo estuvo conformado por 20 ítems, a partir de los cuales se recogió información referente a los niveles de conocimiento en educación ambiental (conceptual, procedimental y actitudinal) y a la disposición de los alumnos hacia las innovaciones en el aprendizaje de la Educación Ambiental (Factibilidad del diseño). La información recogida aportó la información necesaria para diseñar el videojuego propuesto, ajustado a las preferencias y necesidades de los niños y niñas del sexto grado.

\section{RESULTADOS Y DISCUSIÓN}

Una vez aplicado el cuestionario a los sujetos participantes en el estudio, se procedió al análisis de los datos obtenidos, para ello se recurrió a técnicas de estadística descriptiva y al uso de varias representaciones.

La fase del diagnostico reportó, en lo referido a los niveles de conocimiento en educación ambiental (conceptual, procedimental y actitudinal), recogidos en los siguientes ítems:

1. En Venezuela, cuáles de estos animales están en peligro de extinción:

Tonina ___ Oso frontino__

Águila arpía___ Tortuga carey___

Halcón primito ___ Cunaguaro___

Caimán_-_ Guacamaya_.

Anaconda

2. ¿Crees importante cuidar el medio ambiente? Si ___ No___

3. ¿Cuidar el medio ambiente es proteger nuestra vida? Si __ No

4. Se debe promover la protección de los animales y el medio ambiente: $\mathrm{Si}_{-}$No___

5. La educación ambiental ayuda a comprender la importancia de los recursos naturales para la vida en la tierra: $\mathrm{Si}_{-}$No___

Figura 1. Ítems correspondientes a niveles de conocimiento en educación ambiental.

De acuerdo a lo recogido en los ítems 2, 3, 4 y 5 resultados fueron los siguientes (ver tabla 1 ):

TABLA 1. NÚMERO DE ALUMNOS FAVORABLES AL ESTUDIO DE LA EDUCACIÓN AMBIENTAL

\begin{tabular}{|rrr|}
\hline ÍTEM & SI & No \\
\hline 2 & 22 & 0 \\
3 & 22 & 0 \\
4 & 22 & 0 \\
5 & 22 & 0
\end{tabular}

Fuente: Cuestionario aplicado a estudiantes Sexto Grado. 2018 
Los resultados reportaron el interés manifiesto por los alumnos y alumnas hacia los contenidos contemplados en el programa de educación ambiental (Ministerio del Poder Popular para la Educación, 2007). Esto soporta la necesidad de generar mecanismos para fortalecer esta área curricular; en este caso, se haría mediante la incorporación del videojuego como herramienta didáctica en el aula de sexto grado.

En cuanto al ítem número 1, se obtuvo lo mostrado en la figura 2, donde se observa que la mayoría de los alumnos logran identificar algunos animales en peligro de extinción, de acuerdo a los que son reconocidos bajo esta condición en el libro rojo de la fauna venezolana (Rodríguez y Rojas-Suárez, 2015). Sin embargo, es importante acotar que durante la aplicación del cuestionario hubo estudiantes que manifestaron dudas acerca de los riesgos que actualmente tienen algunos de los animales que se sondeaban en el cuestionario.

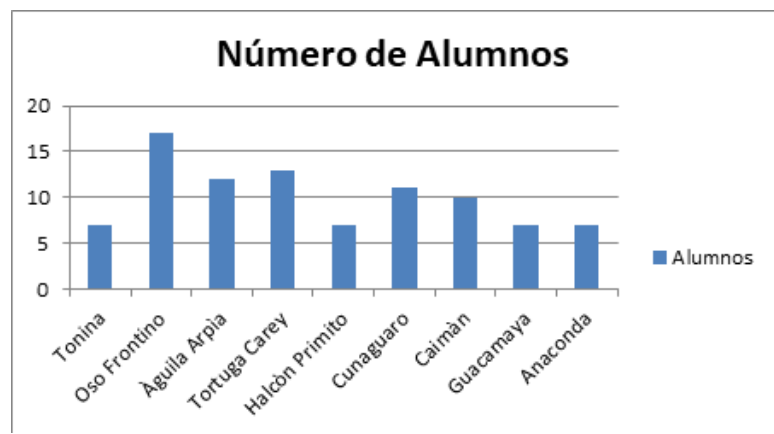

Figura 2: Animales en peligro de extinción, según los alumnos de sexto grado.

Fuente: Cuestionario aplicado a estudiantes Sexto Grado. 2018

En lo referido a la disposición de los alumnos hacia las innovaciones en el aprendizaje de la educación ambiental (ambiente, comunidad y escuela), se presentaron los ítems mostrados en la figura 3.

Los resultados obtenidos en los ítems 6, 7, 8, 9 y 10 se pueden observar en la tabla 2 que se presenta a continuación.

TABLA 2. NÚMERO DE ALUMNOS FAVORABLES AL ESTUDIO DE LA EDUCACIÓN AMBIENTAL

\begin{tabular}{|lll|}
\hline İTEM & SI & NO \\
\hline 6 & 19 & 3 \\
7 & 21 & 1 \\
\hline 8 & 6 & 16 \\
\hline 9 & 9 & 13 \\
10 & 21 & 1 \\
\hline
\end{tabular}

Fuente: Cuestionario aplicado a estudiantes Sexto Grado. 2018
1. ¿Tienes disponibilidad de computadora para videojuegos? Si_ No

2. En la semana, juegas videojuegos en la computadora. Si_ No

En caso afirmativo ¿Cuántas horas a la semana?

3. Los videojuegos son perjudiciales para el estudiante Si_ No

4. Los videojuegos solamente deben jugarse en la escuela Si__ No

5. ¿Los videojuegos ambientales podrían ayudar a conocer más los animales de Venezuela? Si_ No

6. ¿Cuáles son los tipos de videojuegos que tú prefieres?

Laberintos_ Deportivos

Simulación de deportes y otras

situaciones

Estrategias con aventuras gráficas y guerras

7. ¿Cuál es la temática de videojuego que tú prefieres?

Guerra

Aventuras en la naturaleza

Rompecabezas

Vida animal

Experimentos científicos

Historia

Temas generales.

8. Los videojuegos te sirven para:

Mejorar tu autoestima

Mejorar tu memoria

Mejorar la concentración

Para pasar el tiempo

9. Los videojuegos son atractivos porque:

Permiten hacer exploraciones

Tienen imágenes en movimiento

Existe una meta que se debe alcanzar

10. ¿Tienes algún videojuego favorito?
Si
No Cuál?

Figura 3: Ítems correspondientes a la disposición de los alumnos hacia las innovaciones en el aprendizaje de la Educación Ambiental

En la tabla 2 se observa claramente la disposición de los alumnos y alumnas a jugar con videojuegos. De hecho, todos (excepto 1, expresaron que juegan unas 2,5 horas (promedio) a la semana. Por otro lado, la gran mayoría $(72,72 \%)$ consideró que los videojuegos no son perjudiciales para los y las estudiantes. Asimismo, un buen porcentaje de los alumnos y alumnas $(59,09 \%)$, reportaron que los videojuegos se pueden jugar en la escuela y fuera de ella. Por último, casi todos los alumnos y alumnas (excepto 
1) consideraron que los videojuegos ayudarían a conocer más los animales de Venezuela. Los resultados de la tabla 2 , sirven de soporte para generar un ambiente positivo hacia el uso de las innovaciones, en especial el caso particular de los videojuegos, tal como sostiene Vargas (2015).

Respecto a los videojuegos, los alumnos mostraron, en su mayoría, razones del porqué son atractivos para jugarlos (Figura 4). En primer lugar señalan la existencia de una meta a alcanzar, luego las imágenes en movimiento (video) y por último la exploración (ensayo y error). Estas tres características son muy importantes en el proceso de aprendizaje, tal como señalan Ouariachi, Gutiérrez-Pérez y Olvera-Lobo (2017), todo ello en virtud que se logra asociar el alcance de logros a la superación de situaciones que permiten el ensayo y error, y además la dinámica interactiva del videojuego mantiene la atención y el interés en el aprendizaje.

En líneas generales, las respuestas obtenidas en el cuestionario fueron un aporte importante para diagnóstico favorable y orientador para la construcción de la propuesta de videojuegos.

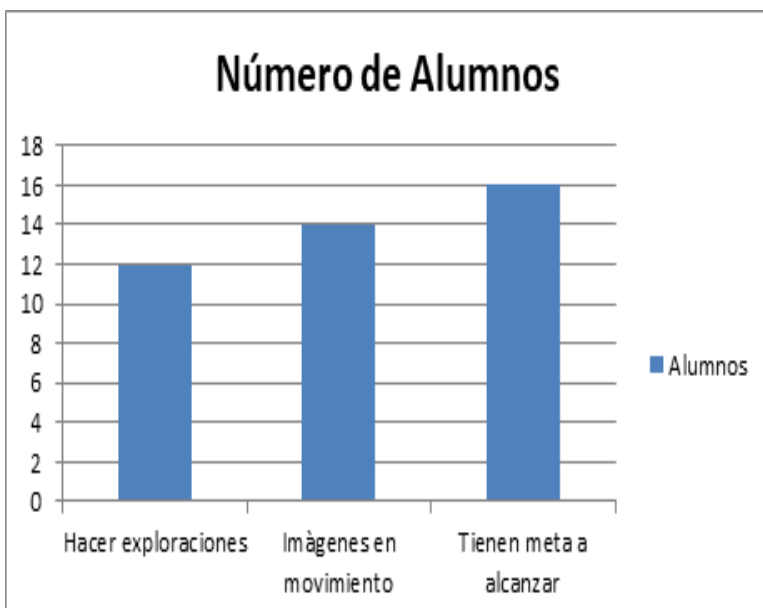

Figura 4. Razones de la atracción de los videojuegos.

Fuente: Cuestionario aplicado a estudiantes Sexto Grado. 2018.

\section{LA PROPUESTA: VIDEOJUEGO MI GRAN AVENTURA POR}

VENEZUELA

La propuesta titulada "Mi gran aventura por Venezuela" constituye un Videojuego educativo (seriousgames), el cual se estructuró tomando en consideración lo evidenciado en las respuestas de los alumnos y alumnas de sexto grado, que fueron encuestados para el diseño del mismo. El videojuego se genera con el propósito que el mismo se constituya en un recurso didáctico para favorecer la comprensión de contenidos en el área de educación ambiental y, más aún, como medio para contribuir concientización de la significación que tiene la existencia de animales en peligro de extinción. En este sentido, con el videojuego los estudiantes efectúen un recorrido virtual por diferentes regiones del país, lo cual les permite conocer e identificar características de sus paisajes y fundamentalmente la fauna en peligro de extinción, de una manera interactiva, divertida y didáctica. El mismo está realizado utilizando el motor gráfico del software UNITY (Creighton, 2010).

La importancia de este recurso, radica en que los alumnos de sexto grado contaran con una herramienta que favorecerá la comprensión de los contenidos en educación ambiental. También será un aporte para cubrir la ausencia de recursos no tradicionales para enseñar y aprender los temas del área ambiental, tal como señalan Colmenares y Sánchez (2015). El videojuego podría llegar, a la mayoría de los alumnos de sexto grado, mediante el sistema de las computadoras Canaima o a través de algún portal educativo.

Con este recurso, los alumnos podrán identificar más animales de nuestra fauna, su hábitat y peligros que ponen en riesgo su existencia en el ecosistema. Los estudiantes tendrán la oportunidad de interactuar con un videojuego educativo y todas las bondades que éste le podría generar. Es decir, este videojuego es de mucha importancia para el subsistema de educación primaria, porque introduce elementos que innovan el aprendizaje y la enseñanza de la educación ambiental, en particular está dirigido a apoyar uno de los objetivos del programa oficial establecido para el sexto grado de educación primaria (Ministerio del Poder Popular para la Educación, 2007), el cuál expresa que el niño debe "Participar en la búsqueda de soluciones a los problemas sociales y ambientales, a fin de formar un ser social integral con conocimientos, experiencias y conciencia ecológica..." (p.16).

Los objetivos de la propuesta (videojuego) son: 1) Facilitar el conocimiento de los componentes de educación ambiental que forman al ciudadano; 2) Promover el respeto por la naturaleza, el conocimiento de sus componentes vitales y el mantenimiento de su estructura; 3) Desarrollar en los alumnos y alumnas un conocimiento amplio y una actitud crítica, responsable y sensible hacia el medio que los rodea.

El videojuego está estructurado en cinco niveles (ver figuras 5 y 6). En cada nivel siempre hay un paisaje, diferente a los presentados en 
los demás niveles. La estrategia en cada nivel se centra en la búsqueda de uno de los animales en peligro de extinción y su hábitat. Se avanza a través de pistas que orientan hacia su ubicación, tomando en cuenta aspectos que lo relacionan.

En la primera versión del videojuego, y tomando en cuenta lo señalado por Rodríguez y Rojas-Suárez (2015), se han incorporado los siguientes animales en extinción: halcón primito (desierto), oso frontino (bosque), tortuga carey (playa), caimán del Orinoco (río) y cachicamo (montaña), los cuales, según este mismo autor, son animales que están "en peligro de extinción por acción de la cacería y por la pérdida de hábitat” (p.103).

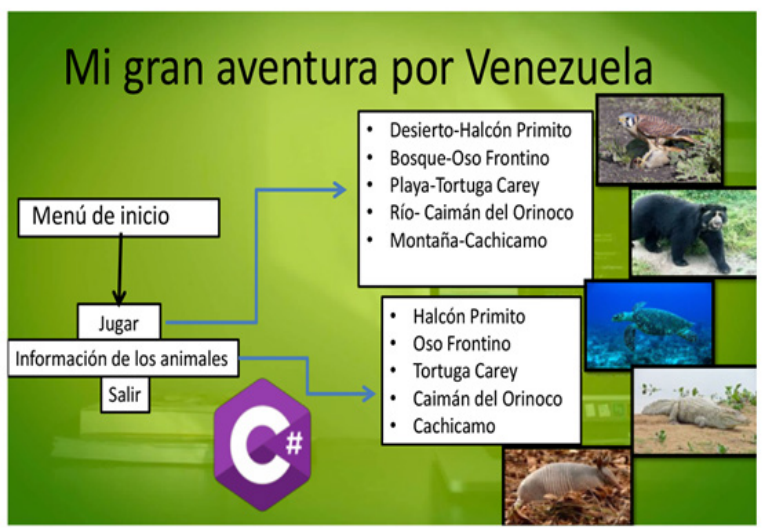

Figura 5: Pantalla estructura del videojuego “Mi gran aventura por Venezuela".

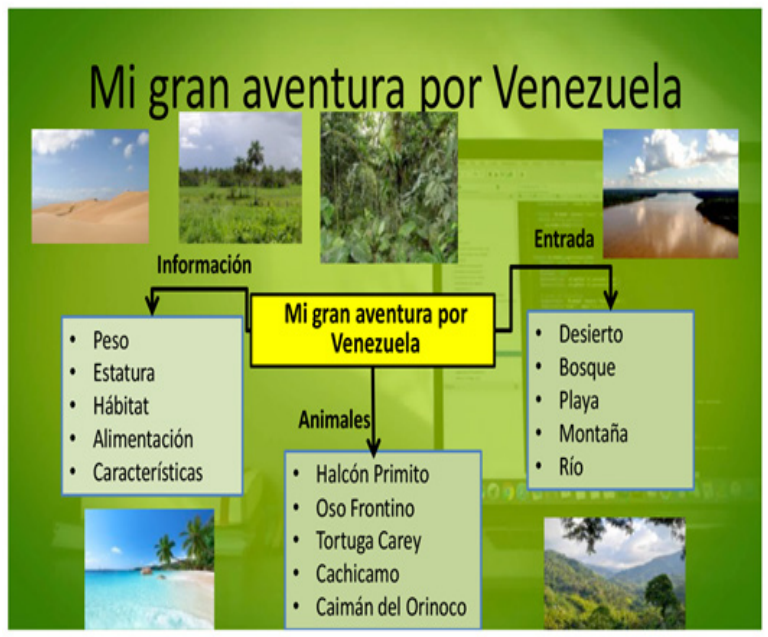

Figura 6: Pantalla componentes del videojuego "Mi gran aventura por Venezuela".

\section{ESPECIFICACIONES TÉCNICAS DEL VIDEOJUEGO “MI GRAN AVENTURA POR VENEZUELA"}

El videojuego propuesto, tiene las especificaciones técnicas que se recogen en el cuadro siguiente:

\begin{tabular}{|c|c|}
\hline REQUISITOS MÍNIMOS & REQUISITOS RECOMENDADOS \\
\hline PROCESADOR INTEL pentium 4 & INTEL ${ }^{\circledR}$ Core 2 Duo FSB800 2.1GH \\
\hline $512 \mathrm{MB}$ de RAM & 2 GB de RAM o superior \\
\hline $\begin{array}{l}\text { Tarjeta gráfica de } 256 \mathrm{MB} \\
\text { Con capacidad de } 800 \times 600\end{array}$ & $\begin{array}{l}\text { Tarjeta grafica de } 512 \mathrm{MB} \text { o superior } \\
\text { Tarjeta de video de una capacidad de } \\
1200 \times 720 \text { o superior }\end{array}$ \\
\hline Sistema operativo Windonws XP SP2 & $\begin{array}{l}\text { Sistema operativo Windows } 70 \\
\text { superior }\end{array}$ \\
\hline Directx 9 RUNTIME & directx 10 o superior \\
\hline $\begin{array}{l}\text { Almacenamiento disponible de } \\
300 \mathrm{Mb}\end{array}$ & Almacenamiento libre de $400 \mathrm{Mb}$ \\
\hline
\end{tabular}

Una de las bondades a resaltar del videojuego es su facilidad de uso; es decir puede ser utilizado en cualquier momento y lugar, siempre que se cumplan las especificaciones dadas. Esta es una gran ventaja que proporciona la tecnología digital.

\section{DINÁMICA DEL VIDEOJUEGO}

El propósito del jugador es rescatar un animal en peligro de extinción. Para dar inicio se muestra una pantalla con varios animales en peligro de extinción, donde el jugador escoge el animal en extinción que desea rescatar. Luego, el jugador inicia el juego y en ese momento empieza la búsqueda. Debe seguir pistas que van apareciendo en el camino contextualizado en el ambiente natural donde viven los animales (médanos, montañas, ríos, bosques, llanura y mar). En la versión actual, el videojuego contempla cinco animales (Halcón primito, cachicamo, oso frontino, caimán del Orinoco y la tortuga carey). Una vez que el jugador rescata un animal en peligro de extinción, le aparecerá una pantalla donde se indican aspectos importantes relacionados con su nombre común y científico, su alimentación, su hábitat, sus aspectos físicos y su forma de reproducción. En otras versiones podrían incorporarse otros animales. El jugador GANA cuando logra rescatar cada uno de los animales. 
CONCLUSIONES

La puesta en práctica de la Propuesta del videojuego es una excelente oportunidad para ampliar las posibilidades de uso de las computadoras Canaima que poseen los estudiantes de las instituciones públicas, así como otras portátiles o PC, a las que podrían tener acceso la mayoría de los estudiantes del subsistema. El videojuego representa una buena posibilidad de uso de las PC en función del propio aprendizaje del estudiante. Este aspecto justifica ampliamente el uso de la propuesta de videojuego que se propone.

Al permitir la exploración virtual de diferentes zonas geográficas del país, se considera que con el uso del videojuego, el estudiante tiene la posibilidad de visitar virtualmente otros lugares geográficos diferentes, distantes a su lugar de residencia, observar sus paisajes, su fauna y su flora. En general, además de la educación ambiental, los niños y niñas podrían adquirir otros conocimientos en ciencias naturales y geografía, abriéndose la posibilidad de ampliar la exploración hacia las matemáticas y otras áreas.

Se espera que con el uso del videojuego los estudiantes de sexto grado, logren adquirir mayor información y conocimiento acerca de los animales en peligro extinción, su hábitat y riesgos de sobrevivencia. En definitiva, los estudiantes que interactúen con el videojuego incrementarán su sensibilidad, conocimientos, actitudes y valores hacia los contenidos curriculares de la educación ambiental; asimismo, se reforzará la conducta conservacionista y de protección hacia la fauna.

Por otro lado, existe la posibilidad que el videojuego, pueda ser de utilidad para muchas escuelas del ámbito regional y nacional, a través de plataformas tecnológicas de instituciones con interés educativo.

Agradecimiento: A los que hicieron posible la presente investigación; de manera muy especial a los niños y niñas del sexto grado, año escolar 2017-2018, de la Escuela Básica Nacional "Aníbal Castillo”, Maracay, Estado Aragua, Venezuela. Asimismo, agradecemos al personal directivo y docente de esta Institución por su apoyo en la fase diagnóstica del estudio.

\section{REFERENCIAS}

Boada, D. \& Escalona, J. (2004). Pedagogía Ambiental en Venezuela y Educación Inicial. Educere, 8(25),181-186. Disponible en: http://www.saber.ula.ve/

Çiftci, S. (2018). Trends of Serious Games Research from 2007 to 2017: A Bibliometric Analysis. Journal of Education and Training Studies, 6(2), 18-27.

Colmenares, K. \& Sánchez, G. (2015). Juego ecológico como herramienta para la formación ambientalista. Trabajo de Grado para obtener el título de Licenciado en Educación mención Educación Integral, en la Facultad de Ciencias de la Educación, Universidad de Carabobo. Disponible en:

Creighton, R.H. (2010). Unity 3D Game Development by Example.Beginner's Guide. Birmingham, Reino Unido: Packt Publishing.

Díaz Barriga, F. (2006). Enseñanza situada: vínculo entre la escuela y la vida. México: McGraw-Hill Interamericana.

Dubs, R. (2002). El proyecto factible. Una modalidad de investigación. SAPIENS. Revista de Investigación Universitaria, 3(2), 1-18.

Escobar, M. (2017). Aprendizaje mediado por videojuegos: potencialidades en el aula y desafíos para la investigación. Revista de Enseñanza de la Física, 29( $\mathrm{N}^{\circ}$ Extra), 519-525.

Gee, J.P. (2003). What video games have to teach us about learning and literacy. Nueva York, USA: Palgrave Macmillan

Grande, M. (2018). Beneficios educativos y videojuegos: revisión de la literatura española. Education in The Knowledge Society (EKS), 19(3), 37-51.

López, M. (2016). El videojuego como herramienta educativa. Posibilidades y problemáticas acerca de los serious games. Apertura. Revista de Innovación Educativa, 8(1),136151.

Malykhina, E. (2014).Fact or Fiction?: Video Games Are the Future of Education. Revista Scientific American. Disponible en:

https://www.scientificamerican.com/ article/fact-or-fiction-video-games-arethe-future-of-education/ Fecha de acceso: $15 / 03 / 2018$

McFarlane, A., Sparrowhawk, A. \& Heald, Y. (2002). Report on the educational use of games. Disponible en: http://www.teem. org.uk/

Fecha de acceso: 26/03/2018 
Michael, D. \& Chen, S. (2006). Serious Games: Games that Educate, Train and Inform. Boston, USA: Thomson.

Ministerio del Poder Popular para la Educación (2007). Currículo del Subsistema de Educación Primaria Bolivariana. Caracas: Autor

Ouariachi, T., Gutiérrez-Pérez, J. y OlveraLobo, M.D. (2017). Criterios de evaluación de juegos en línea sobre cambio climático. Aplicación del método Delphi para su identificación. Revista Mexicana de Investigación Educativa, 22(73), 445-474.

Rodríguez, J. \& Rojas-Suárez, F. (2015). Libro rojo de la fauna venezolana (4ta edición). Caracas: Provita.

Rojo, T. \& Dudu, S. (2017). Los "juegos serios" como instrumento de empoderamiento y aprendizaje socio-laboral Inclusivo. Revista Fuentes. 19(2), 95-109.

Solano, C., Forero, G., Cavanzo, G. \& Pinilla, J. (2013). Concepcioni: videojuego educativo para la enseñanza del proceso de concepción humana. Tecnura, 17(2), 90-99.

UNESCO (1997). Actividades de educación ambientalparalasescuelasprimarias. Santiago, Chile: Autor. Disponible en: http://unesdoc. unesco.org/images/0009/000963/096345so. pdf
UPEL (2016). Manual de trabajos de grado de Especialización, Maestría y Tesis Doctorales (5ta. Edición). Caracas: FEDEUPEL.

Vargas, C. (2015). La creación de videojuegos en ciencias naturales y la competencia para resolver problemas. Revista Lasallista de Investigación, 12(2), 66-74.

Vargas, J. (2016). La mejora del proceso de enseñanza-aprendizaje en educación ambiental. Un estudio centrado en la educación general básica de Ecuador. (Tesis doctoral). Facultad de Educación, Universidad Complutense de Madrid. Disponible en: https://eprints. ucm.es/40504/

Vásquez, M. (2015). El profesorado de ciencias físicas frente al uso de video-juegos comerciales para potenciar el aprendizaje significativo en estudiantes de educación básica superior. Alternativas, 16(3),67-74. Disponible en: http://editorial.ucsg.edu. ec/ojs-alternativas/index.php/alternativasucsg/article/view/91/74 